\title{
A comparison of microtensile and microcompression methods for studying plastic properties of nanocrystalline electrodeposited nickel at different length scales
}

\author{
L. Philippe, ${ }^{\text {a) }}$ P. Schwaller, G. Bürki, and J. Michler \\ EMPA, Swiss Federal Laboratories for Materials Testing and Research, CH-3602 Thun, Switzerland
}

(Received 24 May 2007; accepted 5 February 2008)

\begin{abstract}
A comparison of microcompression and microtensile methods to study mechanical properties of electrodeposited nanocrystalline (nc) nickel has been performed. Microtensile tests that probe a volume of more than $2 \times 10^{6} \mu \mathrm{m}^{3}$ show reasonable agreement with results from microcompression tests that probe much smaller volumes down to a few $\mu \mathrm{m}^{3}$. Differences between the two uniaxial techniques are discussed in terms of measurements errors, probed volume and surface effects, strain rate, and influence of stress state. Uniaxial solicitation in compression mode revealed several advantages for studying stress-strain properties.
\end{abstract}

\section{INTRODUCTION}

In recent years, the LIGA process (combines x-ray lithography with electroplating and plastic molding) for developing structures from nano- to micrometer sizes has become a routine process. ${ }^{1,2}$ The reasons for the success of the galvanoforming method in the microelectromechanical systems (MEMS) industry are numerous and include the fact that metallic pieces with a wellcontrolled microstructure, high aspect ratio, and excellent spatial resolution can be obtained. This opens new horizons in research and industry, with possibilities for changing mechanical properties by tailoring microstructure and alloying elements. For a successful application of such microscopic devices, a detailed understanding of their mechanical properties (at small length scales) is indispensable. ${ }^{3}$ Device design requires the knowledge of the stress-strain behavior of a material over a wide range of strains. ${ }^{4}$ The accurate determination of complete stress-strain curves on the microscopic scale is therefore of vital interest.

Mechanical properties determined with standard, established materials testing procedures at the macroscale cannot be easily extrapolated to smaller length scales. In particular, the errors of the measuring technique are difficult to evaluate if there is a convolution with the socalled extrinsic size effect of the samples. ${ }^{5}$ Along with nanoindentation measurements (which are, however, mainly used for the determination of hardness and Young's modulus), other techniques to test materials at

\footnotetext{
a) Address all correspondence to this author.

e-mail: laetitia.philippe@empa.ch

DOI: $10.1557 / J M R .2008 .0162$
}

micrometer scales are emerging, such as microtensile $e^{6,7}$ and microcompression tests. ${ }^{8,9}$ Size effects in monocrystalline materials were evidenced in the past using mainly nanoindentation and microcompression tests. ${ }^{9,10}$ Although size effects related to internal length scales of materials are widely observed and simulated ${ }^{11,12}$ and used for industrial applications (i.e., improvement of a metal yield strength by grain size refinement), there remains a fundamental challenge to systematically investigate external length-scale effects related to the probed volume in the submillimeter to nanometer regime.

External length-scale effects may be observed at multiple stages over this wide range of sizes, because the mechanisms associated with dislocation storage, multiplication, motion, pinning, and nucleation are generally active over different length scales. ${ }^{5,9}$ To further complicate the size effect, except for instrumented indentation testing there is currently no technique able to probe mechanical properties of the same material at all different sizes; this bring into consideration the volume probed by each different technique used and its potential influence on the measured stress-strain behavior of the material. To evaluate the effects of grain size or geometrical constraints on measured mechanical properties, there is first of all the great need to understand the influence of the measurement technique (load distribution, stress state, etc.) on the measurement values.

One possibility for mechanical testing of small specimens is to scale down conventional testing techniques. Uniaxial tensile testing is in general the most important mechanical testing method. However, downscaling of tensile testing to the submillimeter regime needs to be done very carefully. Many scaling-related artifacts have to be avoided: Geometry measurements need to be done 
with a higher absolute precision in the case of small-scale specimens, and handling of small specimens is more difficult. ${ }^{6,13}$ Gripping devices as well as the alignment of the specimen in the direction of the tensile force are critical. In microcompression experiment, sample preparation assisted by focused ion beam (FIB) is common to test the material at different scales. Surface contamination originating from the preparation can, however, further complicate the interpretation of the measured material properties. ${ }^{14}$

The aim of this work is to make a systematic comparison of uniaxial microtensile and microcompression methods on the same material to get a better understanding of the influence of the volume probed and the method on the mechanical response of the metal. For this task, we used electrodeposited nanocrystalline nickel specimens of the same batch to produce microtensile and microcompression samples. We used nanocrystalline material to minimize extrinsic, e.g., physical sample, effects. We revealed that there is a very good agreement between both uniaxial tests and discuss the errors of variations between both methods, showing a clear interest of compression method when studying highly isotropic materials.

\section{EXPERIMENTAL}

\section{A. Sample fabrication and microstructure characterization}

Nanocrystalline (nc) Ni samples have been manufactured by a LIGA process and were provided by Tecan Ltd., Theale, Dorset, UK. Glow-discharge optical emission spectrometry (GDOES) JY 5000 RF (Horiba Jobin Yvon $\mathrm{GmbH}$, Longjumeau, France) was performed to analyze the elemental composition of the deposit by depth profiling with a resolution down to a few ppm depending on the element (Table I). Samples for tensile and indentation testing having a thickness of $148.75 \pm 0.7 \mu \mathrm{m}$ were produced from the same batch directly in the shape of a conventional, miniaturized tensile test specimen. Roughness $R_{\mathrm{a}}$ of the as-deposited surface was measured by profilometry and was $0.6 \pm 0.05 \mu \mathrm{m}$. By measuring the volume of the deposits and their mass, we could deduce that there was a free volume (pores) of up to $25 \%$ within the matrix. For topography imaging, the sample surface was observed by high-resolution scanning electron microscopy (HRSEM). Transmission electron microscopy (TEM) to determine the grain size (average grain

TABLE I. Elemental composition (wt $\%$ ) of the electrodeposited nickel by means of GDOES measurements. Depth profiling indicates a constant composition over the deposit thickness.

\begin{tabular}{ccccccc}
\hline \hline $\mathrm{Ni}$ & $\mathrm{N}$ & $\mathrm{O}$ & $\mathrm{Si}$ & $\mathrm{C}$ & $\mathrm{H}$ & $\mathrm{S}$ \\
\hline 99.57106 & 0.15798 & 0.12562 & 0.09135 & 0.03933 & 0.0198 & 0.01061 \\
\hline \hline
\end{tabular}

size of $50 \mathrm{~nm}$ with very high aspect ratios, variation range lengths 30-200 nm, see Fig. 1), and x-ray diffraction (XRD) measurements were performed to analyze the crystal structure. XRD using $\Theta-2 \Theta$ scans showed that the materials exhibited a cubic texture. For the microcompression tests, two different types of pillars have been used. Pillars with a height of $30 \mu \mathrm{m}$ and a diameter of $10 \mu \mathrm{m}$ have been directly produced by electrodeposition. FIB machining was used to fabricate smaller pillars, having an average base diameter of $2.47 \mu \mathrm{m}$ and an average top diameter of $1.98 \mu \mathrm{m}$. The average height was $3.76 \mu \mathrm{m}$.

\section{B. Mechanical testing}

In situ scanning electron microscope (SEM) microtensile tests were conducted at room temperature with a tensile stage (Kammrath \& Weiss GmbH, Dortmund, Germany) in a ZEISS DSM 962 SEM. The microtensile $\mathrm{Ni}$ specimens were placed into the gap of a gripping device. The test was conducted under displacement control, and a linear variable differential transducer (LVDT) was used for the measurement of the crosshead displacement. Because of difficulties in gripping and misalignment of the specimen during the test, the strain in the sample was measured via image analysis from the SEM video to ensure a precise measurement. The setup has been adapted for loads up to $15 \mathrm{kN}$. The applied strain rate was $0.0002 \mathrm{~s}^{-1}$. For microcompression loading, we developed a novel method for in situ SEM microcompression testing. ${ }^{15}$

The in situ compression tests were carried out using a diamond flat punch of $15 \mu \mathrm{m}$ diameter. Strain has been calculated from the displacement measurement of the instrumented stack piezo using an instrument compliance

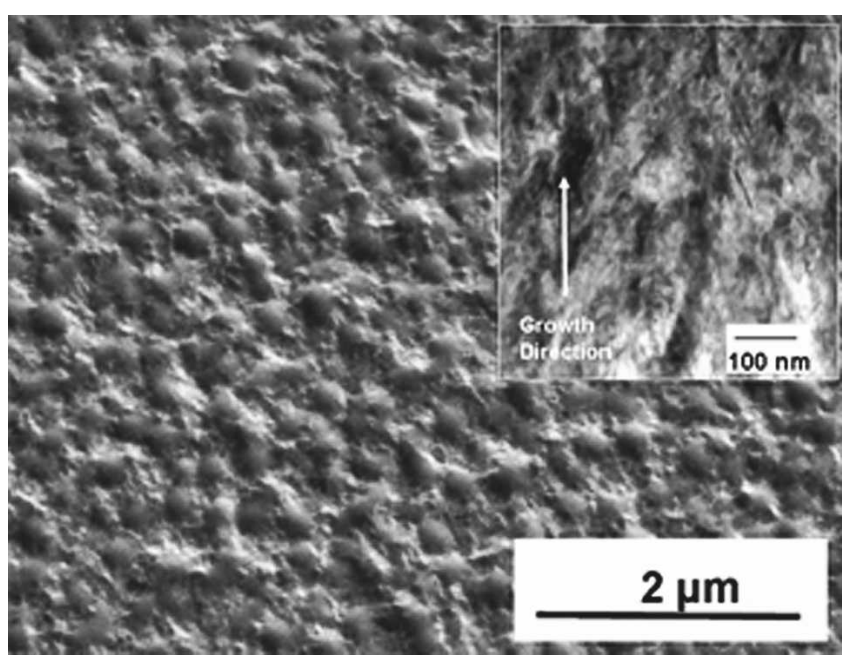

FIG. 1. HRSEM image of the nc Ni surface; roughness and grain size are easily identified on this picture. The inset of the right of the picture shows a TEM bright-field image of the specimen indicating the growth direction of the electrodeposition. 
of $0.0038 \mu \mathrm{m} / \mathrm{mN}$ and corrected for pillar sink-in into the substrate (see Sec. IV. A). To assess the potential of the method, compression tests on the $10-\mu \mathrm{m}$ Ni pillars were performed inside the SEM. In the same way as for tensile measurements, a quantitative evaluation of the deformation during compression was possible through the video frame records. Microcompression tests have the advantage over microbending tests that the sample dimensions can be more easily scaled down to below $1 \mu \mathrm{m}$ diameter. The direct SEM observation during the instrumented compression testing allows very efficient positioning, assessment of the failure mechanisms, and automation of the test. The compression tests were performed using a custom-built instrumented microindentation device that can be used inside the SEM. The instrument, the details of which are described elsewhere, ${ }^{15}$ is based on a load cell fixed on a piezoactuated lateral positioning stage.

Microcompression tests on smaller Ni pillars $(2 \mu \mathrm{m}$ diameter) have been performed in a commercial MTS Nano XP nanoindentation system (MTS System Corporation, Eden Prairie, MN). A truncated tip of $8 \mu \mathrm{m}$ diameter was used, and all tests were conducted at room temperature on FIB-prepared samples.

\section{RESULTS}

Chemical analysis reveals the high purity of the electrodeposited nickel used in the study (see Table I). The compositional depth profiling allows us to conclude that there was homogeneous composition on the entire deposit section. The electrodeposited nc nickel composition is of importance if one wants to avoid impurity segregation (such as sulfur) at the grain boundaries that induces embrittlement.

Figure 1 shows a HRSEM picture of the nc Ni surface; the presence of a rough surface as well as distinction of grains (of size up to $200 \mathrm{~nm}$ ) are clearly visible. The inset in Fig. 1 displays a TEM cross section of the electrodeposited Ni films. The structure is nanocrystalline. The TEM analysis revealed the presence of a predominant columnar microstructure parallel to the deposition direction. At higher magnification, the presence of twins was detected. XRD confirmed the face-centered cubic (fcc) crystal structure.

Figure 2 shows the average true stress-strain $(\sigma-\epsilon)$ curve (i.e., measurements made prior to necking) obtained by microtensile measurements from four different measurements. The curve indicates a weak strain hardening leading to an increase of the required stress for further deformation of the tested specimen. Figure 2(a) shows a SEM image of the dogbone's central section extracted from the video frames recorded at the very beginning of a measurement, and Fig. 2(b) represents the SEM video frame of the microtensile bars just before fracture, revealing necking in the central part. The inset

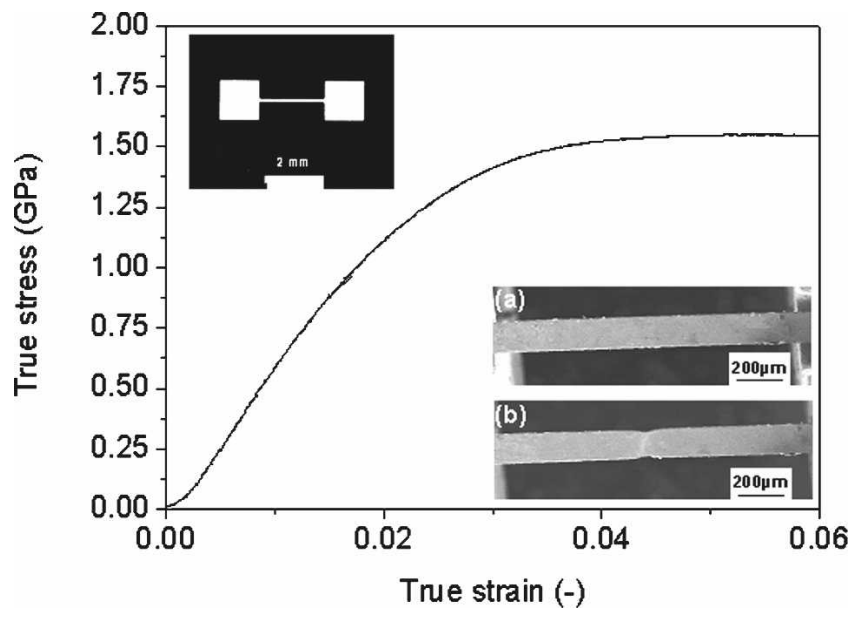

FIG. 2. Average tensile stress-strain curves obtained from four samples. (a) SEM image of the central part of the tensile bar shows video frames at time corresponding to the beginning and (b) the end before fracture of the test. The inset of the left part of the graph shows an optical picture of a typical dogbone specimen tested.

on the left part of the graph shows an optical picture of a typical dogbone used; it shows clearly large extremities of the dogbone to grip and fix the specimen inside a gripping system ensuring a good positioning of the tensile bar.

Figure 3 displays true $\sigma-\epsilon$ curves obtained on $10-\mu \mathrm{m}$ diameter pillars (aspect ratio 3:1) with in situ compression test compared with stress-strain curve obtained on $2-\mu$ m-diameter pillars (aspect ratio 3:2). Few of the $2-\mu \mathrm{m}$ pillars exhibited small off-axis deformation that

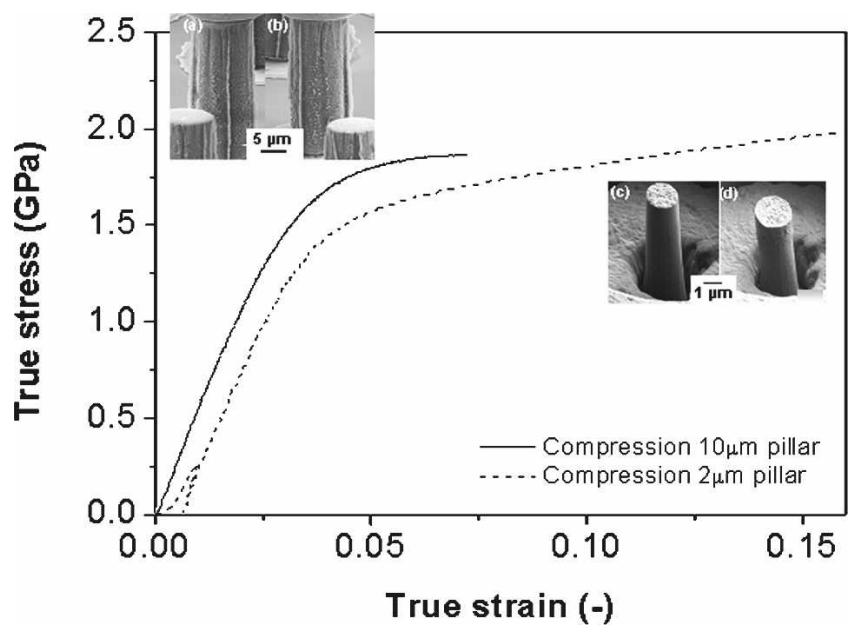

FIG. 3. Average microcompressive stress-strain obtained from five measurements for compression tests on $10-\mu \mathrm{m}$ pillars with in situ microcompression test and on 2- $\mu \mathrm{m}$ pillars for MTS compression tests. On the $2-\mu \mathrm{m}$ pillar curve, a reloading procedure at the beginning of the compression test to rectify the misalignment of the pillar with the tip is visible. In situ SEM image of the pillar made from video frames at time corresponding to (a) beginning and (b) end of the test. Ex situ SEM picture of the FIB pillars (c) before and (d) after microcompression test. A preloading to avoid misalignment is visible on the graph. 
did not influence significantly the measured stress-strain curve. Both curves represent mean true $\sigma-\epsilon$ curves based on five measurements. Figures $3(\mathrm{a})$ and $3(\mathrm{~b})$ for the $10-\mu \mathrm{m}-$ diameter pillar tested represent the video frames extracted at the beginning and at the end of a typical in situ compression test, respectively. Buckling or cracking formation was only rarely found in the video recording, and those were not used for analysis. SEM images Figs. 3(c) and 3(d) were taken ex situ, respectively, before and after the microcompression tests. The sink-in of the pillar into the substrate material is important in this case and has to be accounted for. The reason for this is that the base will always deform along the deformation axis, and this deformation contributes to the system compliance, which is not accounted for when collecting the displacement data. In this work, this error was alleviated by accounting for the compliance through the calculation of the base compliance. By subtracting this base compliance from the total compliance, a corrected stiffness (and hence modulus) is obtained as proposed by Zhang et al. ${ }^{13,16}$

Table II lists the respective material properties inferred from $\sigma-\epsilon$ curves obtained with the different testing methods. The yield stress values listed in Table II are equal to the $0.2 \%$ offset yield stress (i.e., a line shifted by a strain of 0.002 is drawn parallel to the linear elastic part; the intersection of this line with the stress-strain curve is taken as yield stress $\sigma_{\mathrm{y}}$ ) commonly used in materials technology, and the Young's modulus is derived from the slope of the $\sigma-\epsilon$ curve in the elastic range.

Figure 4 makes a comparison between the stress-strain curves obtained with microcompression and microtensile measurements. On each curve, the probed volume of the technique is indicated. From Table II, we note a slightly lower value of the Young's modulus assessed in compression modes.

\section{DISCUSSION}

The complete stress-strain curves determined by three different methods reveal that there is a rather fair agreement between the two compression and the tensile tests despite the fact that the probed volume differs by five orders of magnitude. In this section, we discuss these experimental result discrepancies in terms of measurement errors, probed volume and surface effects, strain rate, and influence on stress state.

TABLE II. Material properties (Young's modulus, $E$; yield stress, $\sigma_{\mathrm{y}}$ ) for $\mathrm{Ni}$ as determined from microtensile and compression testing. Comparison of the strain rates values used for each method.

\begin{tabular}{lccc}
\hline \hline \multicolumn{1}{c}{ Method } & $E(\mathrm{GPa})$ & $\sigma_{\mathrm{y}}(\mathrm{GPa})$ & Strain rates $\left(\mathrm{s}^{-1}\right)$ \\
\hline Tensile & $63 \pm 16$ & $1.2 \pm 0.1$ & $0.2 \times 10^{-3}$ \\
Compression (2- $\mu \mathrm{m}$ pillar) & $51 \pm 13$ & $1.3 \pm 0.2$ & $0.9 \times 10^{-3}$ \\
Compression $(10-\mu \mathrm{m}$ pillar) & $54 \pm 14$ & $1.4 \pm 0.2$ & $1.5 \times 10^{-3}$ \\
\hline \hline
\end{tabular}

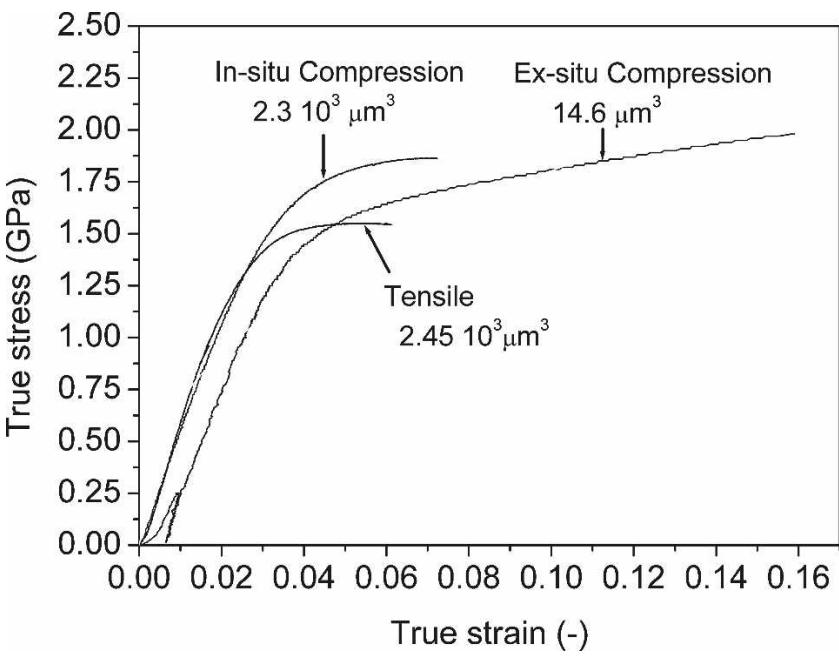

FIG. 4. Comparison of the stress-strain curves obtained for the different mechanical testing methods on nc electrodeposited nickel: in situ microcompression, ex situ microcompression, and microtensile tests. For each experiment, the probed volume is labeled.

\section{A. Measurement errors}

Measurement errors that are intrinsic to each technique are prone to give differences in $\sigma-\epsilon$ curves.

For tensile tests, the major source of uncertainty in the stress calculation being the measurement of the crosssectional area of the specimen, 20 microtensile bars from the same batch as the ones tested were embedded in resin and polished to their cross section. The measured optical image area for each cross section and an average value of the tensile bars cross section were calculated. As all tensile bars tested are issued from the same plating batch, we assume that an average value of their cross sections gives sufficient accuracy.

For a compression test, the pillar shape is crucial. The $10-\mu \mathrm{m}$ pillar cross section was determined by measurements on pillars that were embedded in resin and polished to their cross section. Pillars cross section were measured and then repolished again for another section measurement over its entire length until their basement, to make 10 consecutive measurements of the pillar cross section over its entire length. For $2-\mu \mathrm{m}$ pillars, 10 measurements of the cross section were made directly on the pillar SEM image and over its entire length for getting an average value of the pillar volume; i.e., the pillar shape being a source of error as the diameter is not constant over the length.

In both cases, for microcompression and microtensile tests, the pillar sizes (length $L$ and surface area $A$ ) or tensile bar sizes (length $L$, and cross section $A$ ) can be measured with an accuracy of $5 \%$ for $L$, and $L_{0}$ (displacement) and with an error of $15 \%$ for $A$. This induces for these statistical errors a scatter of $25 \%$ in the Young's modulus estimation and of $15 \%$ for $\sigma_{\mathrm{y}}$. Such experimental errors on the measurements might explain by themselves 
the discrepancies found between tensile and compression tests.

In the microcompression experiments, FIB samples of $2 \mu \mathrm{m}$ diameters and electrodeposited $10-\mu \mathrm{m}$ pillars are compared. In this way, probed volume and surface conditions are simultaneously changed, which leads to difficulty in comparing the data. We estimate that roughness of the FIB pillar is the same as those of tensile and electrodeposited pillars samples as it was cut out of an electrodeposited nickel sample. However, surface conditions are clearly changing from the FIB processing, and recent results suggest that this cannot be neglected. ${ }^{14}$ Furthermore, pillar asymmetry and texture effect is a problem as there is not a compression test on the whole deposited volume.

This comparison between microcompression tests on different pillar sizes and using two different setups shows that it is possible to obtain mechanical properties of mechanically isotropic materials to very good accuracy with microcompression experiments. However, for tests using conventional nanoindentation equipment with a flatended tip, special care has to be taken. First, the measured displacement values have to be corrected by accounting for the sink-in of the pillar into the substrate, whereas this correction is not necessary when strain values are determined from SEM images recorded during compression. Also, this correction has to take into account the complex geometry of the postbase connection if one wants to avoid an overestimation of the Young's modulus with a simple elastic contact model. ${ }^{16}$ Finally, without a continuous video control, a misalignment of the tip to the pillar can lead to a decrease of the measured elastic modulus or in excessive cases to the buckling of the pillars.

Overall, the difference found in the results between the three methods (Table II and Fig. 4) can be yet explained by the experimental errors described in this subsection. However, other contributing effects to the differences observed between the measurements are reviewed below.

\section{B. Probed volume and surface effects}

The probed volume is different for each technique, as shown in Fig. 4. Even though the number of probed grains varies over several orders of magnitudes for the different experimental setups, compared with the grain size the deformed volumes are large in either case. Thus, the difference of probed volume in the different tests, i.e., external size effects, should not have a significant influence on the $\sigma-\epsilon$ curves. However, the smaller the volume, the more important the surface of an object gets, and one has to ensure that the surface region does not have significantly different properties than in the bulk. For instance, a thin native oxide layer (typically of around $50-300 \AA$ for $\mathrm{Ni}^{17}$ ) or an ion implantation zone becomes more important at smaller samples sizes.
Young's modulus values and yield stresses are somewhat lower than already reported for fully densified nc electrodeposited nickel, ${ }^{5}$ suggesting that the influence of textures, pre-existing voids, columnar grain structure, and hydrogen on the mechanical response cannot be neglected. ${ }^{4,18}$

For the uniaxial tests, (not true for the $2-\mu \mathrm{m}$ pillars because the entire electrodeposited volume is not tested) where measurements on the whole electrodeposited volume are performed, there should be a moderated texture effect influence on the calculated Young's modulus values. Indeed, Table II shows that differences between tension and compression Young's modulus values obtained are little; differences are related to the grain orientation differences between the two methods and to the fact that free volume (pores and flaws) within the matrix tested are prone to play a bigger role on the mechanical response in tensile mode.

Finally, it is important to look not only at the average grain size $(\sim 50 \mathrm{~nm})$, but also the size of the longest grain (up to $200 \mathrm{~nm}$ ) as observed by TEM and its shape (not circular but having a high aspect ratio). This is important because a few large grains can dominate the deformation behavior of the material and determine its performance. ${ }^{19,20}$ Again, this will not have the same consequence depending on the nature of the stress direction applied; in compression measurement the stress is applied parallel to the grain length, whereas in tensile tests the stress is perpendicular to it. The mechanism of deformation and the properties of the nc material not only depend on the average grain size, but are strongly influenced by the grain size distribution, the grain aspect ratios, and the grain boundary structure. ${ }^{4}$

\section{Strain rate}

The strain-rate sensitivity of fcc nc metals is already well reported in literature, ${ }^{4,21,22}$ where it was found that nc pure nickel exhibits a positive strain-rate sensitivity in flow stress. ${ }^{21}$ In this study, variation between the strain rates values for the two methods is not significant (between $0.2 \times 10^{-3} \mathrm{~s}^{-1}$ and $1.5 \times 10^{-3} \mathrm{~s}^{-1}$ ), and loaddisplacement curves for the different strain values did not show a significant influence on the measurement (see Table II). Yield stress value is expected to increase with increasing strain rate ${ }^{4,21}$ that goes in the right direction to the present measurements (i.e., higher yield stresses values obtained in compressive mode). However, models have been developed for grain sizes smaller than $50 \mathrm{~nm}$, but a mechanistic rationalization for the influence of strain rate sensitivity in nc fcc metals becomes less straightforward for large grain sizes. ${ }^{23}$ Therefore, a complete picture that explains the more-or-less continuous decrease in strainrate sensitivity, or alternatively the increase in activation volume with increasing grain size will require additional analysis that goes beyond this work. 


\section{Influence on stress state}

Table II and Fig. 4 show, that tensile and compression tests give $\sigma-\epsilon$ curves and material properties that are in relatively good agreement to each other. Cheng et al. proposed a deformation mechanism map for fcc metals. ${ }^{21}$ The model predicts not only the strength as a function of grain size, but also the observed tension/microcompression asymmetry of the yield strength. It is predicted that a higher yield stress will be found in compression than in tension for fcc metals with grain sizes ranging from 2 up to $100 \mathrm{~nm}$. The tension/compression asymmetry, which is explained by a pressure dependence of the dislocation self-energy during bow-out, may be responsible for the difference in yield stress values plotted in Table II for the tested fcc nc nickel.

\section{v. CONCLUSION}

We assessed the potential of microcompression and microtensile methods to characterize nanocrystalline metals and discussed extensively potential measurement errors. These measurement errors explain alone the slight variation in the mechanical properties assessed by the two methods. Because of the small grain, size effects were neglected, which revealed on one hand the importance of load cases particular to each method, and on the other hand the influence of the probed volume and of the microstructure. The importance of surface effects, stress state, and strain rate could not be distinguished. We found that uniaxial tensile and uniaxial compression testing is able to provide accurate data and similar mechanical insights of the tested materials of five orders of magnitude of probed volume. Experimental validation of emerging strain-gradient based continuum theories of deformation (models that incorporate a physical length scale into the constitutive relations for the mechanical response of materials) must carefully account for these experimental issues that extend beyond the gradientinduced storage of defects. Comparing the aforementioned advantages and drawbacks of the different approaches one may conclude that in this context microcompression tests are very appropriate to study mechanical properties of isotropic nc metals.

\section{REFERENCES}

1. Y. Yang, B.I. Imasogie, S.M. Allameh, B. Boyce, K. Lian, J. Lou, and W.O. Soboyejo: Mechanisms of fatigue in LIGA Ni MEMS thin films. Mater. Sci. Eng., A 444, 39 (2007).

2. B. Li and Q. Chen: Solid micromechanical valves fabricated with in situ UV-LIGA assembled nickel. Sens. Actuators, A 126, 187 (2006).

3. N.R. Moody, J.M. Jungk, M.S. Kennedy, S.V. Prasad, D.F. Bahr, and W.W. Gerberich: Mechanical properties of wear tested LIGA nickel, in Fundamentals of Nanoindentation and Nanotribology III, edited by K.J. Wahl, N. Huber, A.B. Mann, D.F. Bahr, and
Y-T. Cheng (Mater. Res. Soc. Symp. Proc. 841, Warrendale, PA, 2005), R7.8.

4. K.S. Kumar, H. Van Swygenhoven, and S. Suresh: Mechanical behaviour of nanocrystalline metals and alloys. Acta Mater. 51, 5743 (2003).

5. W.D. Nix, J.R. Greer, G. Feng, and E.T. Lilleodden: Deformation at the nanometer and micrometer length scales: Effects of strain gradients and dislocation starvation. Thin Solid Films 515, 3152 (2007).

6. E. Mazza, S. Abel, and J. Dual: Experimental determination of mechanical properties of $\mathrm{Ni}$ and $\mathrm{Ni}-\mathrm{Fe}$ microbars. Microsyst. Technol. 2, 197 (1996).

7. M.A. Haque and M.T.A. Saif: In-situ tensile testing of nanoscale specimens in SEM and TEM. Exp. Mech. 42(1), 123128 (2001).

8. B. Moser, R. Schwaiger, and M. Dao: Size effects on deformation and fracture on nanostructured materials, in Nanostructured Coating, edited by A. Cavaleiro and J.Th.M. De Hosson, Nanostructure Science and Technology Series (Springer, New York, 2006).

9. J.R. Greer, W.C. Oliver, and W.D. Nix: Size dependence of mechanical properties of gold at the micron scale in the absence of strain gradients. Acta Mater. 53, 1821 (2005).

10. M.D. Uchic, D.M. Dimiduk, J.N. Florando, and W.D. Nix: Sample dimensions influence strength and crystal plasticity. Science 305, 986 (2004).

11. H.D. Espinosa, M. Panico, S. Berbenni, and K.W. Schwartz: Discrete dislocation dynamics simulations to interpret plasticity size and surface effects in freestanding fcc thin films. Int. J. Plast. 22, 2091 (2006).

12. W.D. Nix and H. Gao: Indentation size effects in crystalline materials: a law for strain gradient plasticity. J. Mech. Phys. Solids 46, 411 (1998).

13. I.N. Sneddon: The relation between load and penetration in the axisymmetric Boussineq for a punch of arbitrary profile. Int. J. Eng. Sci. 3, 47 (1965).

14. H. Bei, S. Shim, M.K. Miller, G.R. Pharr, and E.P. George: Effects of focused-ion-beam milling on the nanomechanical behavior of a molybdenum-alloy single crystal. APL 91, 111915 (2007).

15. B. Moser, K. Wasmer, L. Barbieri, and J. Michler: Strength and fracture of Si micropillars: A new scanning electron microscopybased micro-compression test. J. Mater. Res. 22(4), 1004 (2007).

16. H. Zhang, B.E. Schuster, Q. Wie, and K.T. Ramesh: The design of accurate micro-compression experiments. Scripta Mater. 54, 181 (2006).

17. W.F. Mohamad, A. Abou Ajar, and A.N. Saleh: Effects of oxide layers and metals on photoelectric and optical properties of Schottky barrier photodetector. Renew. Ener. 31, 1493 (2006).

18. R.J. Asaro and S. Suresh: Mechanistic models for the activation volume and rate sensitivity in metals with nanocrystalline grains and nano-scale twins. Acta Mater. 53, 3369 (2005).

19. V.L. Tellkamp, A. Melmed, and E.J. Lavernia: Grain growth behavior of a nanostructured $5083 \mathrm{Al}-\mathrm{Mg}$ alloy. Metal. Mater. Trans. A 32, 2335 (2001).

20. T. Hanlon, Y-N. Kwon, and S. Suresh: Grain size effects on the fatigue response of nanocrystalline metals. Scripta Mater. 49, 675 (2003).

21. S. Cheng, J.A. Spencer, and W.W. Milligan: Strength and tension/ compression asymmetry in nanostructured and ultrafine-grain metals. Acta Mater. 51, 4505 (2003).

22. J.L. Loubet, M. Bauer, A. Tonck, S. Bec, and B. Gauthier-Manuel: Mechanical Properties and Deformation Behaviour of Materials Having Ultra-fine Microstructures (Kluwer Academic Publishers, The Netherlands, 1993), pp. 429-447.

23. T. Chudoba, P. Schwaller, R. Rabe, J-M. Breguet, and J. Michler: Comparison of nanoindentation results obtained with Berkovich and cube-corner indenters. Philos. Mag. 86(33-35), 5265 (2006). 